\title{
LA INVESTIGACIÓN COMOESTRATEGIA DE ENSEÑANZA DE LAS CIENCIAS NATURALES: CONCEPCIONES PEDAGÓGICAS DE LOS DOCENTES DEEDUCACIÓN MEDIA EN EL INSTITUTO PEDAGÓGICO NACIONAL
}

\section{The Research as Natural Sciences Teaching Strategy: Pedagogical Conceptions of Secondary Education Teachers at Instituto Pedagógico Nacional}

\author{
Dayana Milena Bejarano Muñoz ${ }^{1}$
}

Fecha de recepción: 2 de marzo de 2015

Fecha de aprobación: 6 de junio de 2015

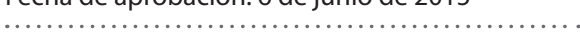

\section{Resumen}

Este trabajo constituye una mirada hacia la investigación como eje de transformación y generación de conocimiento entre los estudiantes de educación media, a partir del análisis de las concepciones pedagógicas de los docentes del Instituto Pedagógico Nacional, en torno a la investigación y la enseñanza de las ciencias naturales. Se implementó una metodología cualitativa desde el enfoque interpretativo, que permitió, desde el estudio de caso, establecer cuáles son las concepciones pedagógicas de los docentes de educación media en el área de ciencias naturales frente a la investigación. Además de ello, se plantean elementos pedagógicos frente a la inclusión de la investigación escolar en la educación media como estrategia de enseñanza de las ciencias naturales, lo cual aporta a la construcción y la transformación de experiencias pedagógicas.

A manera de conclusión, la tendencia de las concepciones de los docentes se vio enfocada hacia la corriente positivista, la cual se enmarca dentro de investigaciones disciplinares y cuantitativas que ven la ciencia desde la aplicación del método científico; aunque se empieza a incluir la corriente pedagógica interpretativa y crítico-social, al socializar los hallazgos cuantitativos obtenidos procurando generar transformaciones sociales desde la intervención con la comunidad. Así mismo, los docentes reconocen los beneficios académicos, sociales, interpersonales y laborales que se obtienen en un proceso de investigación, como la generación y la profundización del conocimiento, el seguimiento de procesos metódicos en la búsqueda de información y recolección de datos, la interpretación y la argumentación frente a fenómenos y el desarrollo crítico desde su cotidianidad; todo lo cual lleva al estudiante a ser un actor de procesos de transformación desde su interés.

Palabras clave: concepciones pedagógicas, investigación, enseñanza de las ciencias naturales, educación media

1 Licenciada en Biología, Universidad Pedagógica Nacional. Docente de educación básica y media, Instituto Pedagógico Nacional. Correo electrónico: dayanambm5@gmail.com. Apartado de la investigación realizada como trabajo de grado para optar al título de Magíster en Educación, Universidad Santo Tomás, Bogotá, D. C., Colombia, 2014. 


\section{Abstract}

This text is a look to the research as a transformation and generation axis of knowledge among middle school students, based on the analysis of teachers' pedagogical conceptions at Instituto Pedagógico Nacional around natural sciences research and teaching. A qualitative methodology from the interpretive approach was implemented, which allowed, from case study, to establish pedagogical conceptions of secondary education teachers in natural sciences about research. In addition, pedagogical elements are proposed about inclusion of school research in secondary education as natural sciences teaching strategy, which contributes to the construction and transformation of educational experiences.

As a conclusion, teachers' trend of conceptions was towards positivism, which is part of disciplinary and quantitative researches, looking at science from the application of scientific method. Even though, pedagogical interpretive and critical-social current begins to be included, by socializing quantitative findings obtained generating social changes from the intervention with the community. Likewise, teachers recognize the academic, social, interpersonal and working benefits obtained in a research process, such as generating and deepening of knowledge, monitoring of methodical processes in search of information and data collection, interpretation and reasoning about phenomena, and critical development from their daily lives, all leading students to be actors of transformation processes from their own interest.

Keywords: pedagogical concepts, research, natural sciences teaching, secondary education

\section{Introducción}

La investigación debe ser un medio de transformación que se incluya en la educación primaria y media, desde edades muy tempranas, pues relegarla a la educación superior genera desconocimiento de las habilidades innatas de los niños y los jóvenes como seres curiosos, inquietos de su realidad y activos en su entorno. Pero para profundizar en un desarrollo de la investigación en la escuela, se deben tener en cuenta la formación y las concepciones de los docentes en torno a ella, pues es importante enfatizar en cómo estos logran desde la transposición didáctica ejercer, guiar y motivar prácticas investigativas en sus estudiantes.

La investigación incentiva en los estudiantes la idea de ser creadores e innovadores, y así se convierte en una forma de construcción de nuevas realidades sociales, donde ellos son actores de su entorno. Esto, pensando como docentes, permite también generar nuevos espacios de aprendizaje, que posicionen al maestro como investigador y guía de procesos investigativos; por ello, es necesario retomar las concepciones desde la definición de Carvajal y Gómez (citados por Aldana, 2008) como construcciones que se han dado a partir de las vivencias, la formación académica, el desempeño profesional, la reflexión sistemática y colectiva, que se materializa en la práctica docente, en la cual, notablemente, influyen factores psicológicos, antropológicos, culturales, sociales, políticos e ideológicos. Esto permite tener en cuenta la manera como estos relacionan sus referentes pedagógicos con su práctica y la incidencia que ello tiene sobre la formación de sus estudiantes; además, permite destacar la importancia de la capacitación permanente, la formación académica e investigativa de los maestros.

De igual forma, Gimeno (citado por Cañal, 2007) define al docente como el facilitador del aprendizaje y como investigador del aprendizaje del aula; por ello, es tan importante establecer cuáles son las miradas, los fundamentos, las estrategias pedagógicas, los conocimientos disciplinares y las prácticas investigativas que este ejerce, según el estudio de sus concepciones frente a investigación.

En la escuela también se ha pensado la investigación, debido a los motivantes que surgen en los estudiantes a partir de sus propias inquietudes y de una cultura de la búsqueda, el cuestionamiento y la indagación de la información y de los fenómenos que los rodean; sin embargo, es allí donde más se limita y se ha demeritado, bajo los interrogantes: ¿Se puede investigar en la escuela? ¿Qué se puede investigar? ¿Pueden llegar a investigar los estudiantes? Aparece así una disyuntiva entre el rol del docente, los intereses de los estudiantes y las normativas de la educación. Por todo eso, la investigación debe ser reconocida como:

\footnotetext{
Una actividad propia del ser humano, posible de desarrollar en todas las áreas del conocimiento y con niños y niñas muy pequeños. También es entendida como un proceso de desciframiento de la condición humana, a partir de la experiencia de vida de esta población en los contextos escolares, familiares y comunitarios. (Colciencias, 2012, p. 23).
}

Lo anterior permite entender la importancia de la investigación en la formación en todos los ámbitos educativos, así como su impacto en el desarrollo individual y social.

Es así como resulta importante incluir la investigación en la escuela media, teniendo en cuenta las concepciones que los maestros manejan en torno a ella, pues sus referentes epistemológicos, pedagógicos y disciplinares son los que fundamentan sus prácticas en el aula y el proceso de enseñanza-aprendizaje de sus estudiantes. 
Profundizando aún más, desde la investigación escolar se pueden retomar diferentes ejes y contenidos temáticos por trabajar en los planes de estudio de las áreas; en especial, en ciencias naturales. La experimentación, las relaciones con la cotidianidad y el aprendizaje significativo han enmarcado los intereses de la enseñanza de las ciencias naturales buscando la trasposición didáctica de los maestros desde sus conocimientos a prácticas en el aula. Pensar en la investigación escolar como estrategia de enseñanza y aprendizaje podría acercar a explorar las capacidades del estudiante, a entender el mundo en el que vive; ello, valiéndose de la capacidad innata de este de preguntarse y de construir su propio conocimiento y desarrollar o potenciar actitudes frente a su entorno.

Además de lo anterior, se hace pertinente ampliar la búsqueda en las concepciones de los maestros, pues a partir de sus conocimientos, sus prácticas, sus estrategias y sus vivencias, ellos enmarcan el proceso de aprendizaje de la investigación en los escolares. Teniendo en cuenta estos parámetros, un acercamiento a la inclusión de la investigación es el desarrollado por el Instituto Pedagógico Nacional (en adelante, IPN), el cual se concibe como un ente académico-administrativo de la Universidad Pedagógica Nacional (en adelante, upN), y, por ende, es un escenario de innovación e investigación pedagógicas. Allí se ha establecido un trabajo en torno a la investigación en varios campos: uno de ellos, en escolares, y el cual busca fortalecer su formación desde el desarrollo de competencias investigativas, por parte de los docentes, desde su quehacer cotidiano, y por parte de los profesores en formación de las universidades que ejercen su práctica pedagógica.

Sin embargo, en estos desarrollos los docentes, como actores principales, deben mantenerse en continua actualización, dado que en esta profesión nunca se dejará de aprender; dicha actualización permitiría la mejora del quehacer pedagógico, lo cual se suma a la importancia de la investigación, por cuanto estos procesos de comprensión, de análisis, de interpretación y de descripción necesitan rigurosidad y compromiso, lo que les permite llegar llevar a la reflexión y a la trasformación de las prácticas educativas y sociales. Marín (2012) sostiene que "la investigación es la base de la formación docente y de las prácticas pedagógicas" (p. 11), y recalca la necesidad y la importancia del docente, no solo como facilitador del conocimiento, sino también, como el investigador de su propia práctica y de su propia actividad profesional y disciplinar.

De esta manera, se plantea como objetivo investigativo establecer las concepciones pedagógicas de los docentes de educación media del IPN sobre investigación, para aportar elementos pedagógicos específicos que permitan abordar la enseñanza de las ciencias naturales. En esa medida, al hablar de concepciones se debe dirigir la mirada a la formación docente, la cual, como lo define Díaz (citado por Gorodokin, 2005),

es más que una sumatoria de conocimientos adquirida por el alumno ya que estructura representaciones, identificaciones, métodos y actitudes e impacta en el sujeto en formación en el plano cognoscitivo, y en lo socio-afectivo, conformando cambios cualitativos más o menos profundos. (Gorodokin, 2005, p. 4).

Por ello, se retomaron como corrientes pedagógicas, en los términos de Herrán, Hashimoto y Machado (2005), la positivista, la interpretativa y la disciplinar. Desde el enfoque positivista, se identificaron en las prácticas docentes características que buscaban construir una propuesta universal a partir de datos; la ciencia es vista desde hechos corroborables y enmarcados dentro de un proceso detallado y estadístico, desde el análisis objetivo de un fenómeno. Desde el enfoque interpretativo, los docentes describen procesos de interacción en el aula y construcción social de los sentidos, incluyendo aspectos culturales y comportamentales y reflexiones cualitativas; por último, retoman la ciencia como la aplicación estricta de una disciplina. Tamayo (1987) plantea que la enseñanza de la investigación se ha orientado al desarrollo de la estructura formal, la estructura epistemológica y la estructura de procesos, por lo cual en la escuela se puede adoptar desde diferentes miradas, tanto en la construcción escrita de documentos que den cuenta del proceso investigativo, del análisis de fundamentos en torno a métodos y aplicaciones, y del seguimiento riguroso de procesos experimentales y de búsqueda y corroboración de hipótesis.

La enseñanza de la ciencia no puede pasar por alto el conocimiento empírico en la construcción del conocimiento científico; así mismo, debe incluir el conocimiento filosófico en las relaciones de los saberes, donde se plantea la investigación como un proceso que direccione dicha construcción. Abordar de forma compleja el conocimiento desde diferentes miradas, no solo la científica, permite reconocer el valor del conocimiento a partir de otras experiencias.

En torno a los estándares en educación, el Ministerio de Educación Nacional (MEN) contempla "la institución escolar como aquella que desempeña un papel privilegiado en la motivación y en el fomento del espíritu investigativo innato de cada estudiante y por ello puede constituirse en un "laboratorio" para formar científicos naturales y sociales" (MEN, 2012, p 34), por lo cual es conveniente que la investigación sea trabajada en el ámbito escolar desde lineamientos y enfoques claros, que le permitan al estudiante acercarse a la búsqueda rigurosa, metódica, descriptiva, 
interpretativa y comprensiva de fenómenos sociales, que le permitan ser actor y crítico de trasformaciones sociales.

Este trabajo comprende un acercamiento a los alcances de la investigación escolar en la formación de estudiantes frente al manejo de la información, la redacción y la escritura, pero, sobre todo, en su formación científica, en la necesidad de su desarrollo en las instituciones educativas como eje de fortalecimiento y acercamiento a la ciencia y el reconocimiento a la influencia del ejercicio docente en la transformación de la sociedad, y señalando la importancia de su formación permanente en el campo de la investigación. Además de ello, se aterriza al contexto del IPN, como centro de práctica e investigación educativa de la UPN, y el cual es un escenario privilegiado en el fortalecimiento de estrategias que derivan de la innovación educativa y del constante ejercicio de investigación pedagógica. Por esta razón, y buscando la manera de entender cómo los docentes ejercen y enseñan la investigación en el IPN desde su formación y su ejercicio, como estrategia de construcción de conocimiento en torno a las ciencias naturales y de transformación de la práctica educativa, se plantea la siguiente pregunta: ¿Cuáles son las concepciones pedagógicas que los docentes de educación media del IPN tienen en torno a la investigación, desde la enseñanza de las ciencias naturales?

\section{Metodología}

Para esta investigación se adopta el enfoque cualitativo, pues se buscó una interpretación del fenómeno en la educación media en el IPN desde el análisis de las concepciones de los docentes, con una visión que permitió que el investigador, a partir del contexto natural, construyera conocimiento en la cotidianidad del ejercicio docente. Además de ello, a partir de las características de los estudios cualitativos planteados por Stake (2005), esta investigación se presenta como eje contextualizado de una realidad a partir de su comprensión, para el caso las concepciones docentes, abordando los diferentes actores del proceso desde su desarrollo investigativo en ciencias naturales; es un trabajo interpretativo desde la interacción con la población sujeto de estudio que se evidencia desde los docentes, y empático, por cuanto busca la información retomando a los actores como marco de referencia; son los docentes quienes abordan la investigación en la enseñanza de las ciencias naturales en la educación media.

Se desarrolla esta investigación dentro del marco de la hermenéutica, bajo la mirada de Dilthey (citado por Briones, 2002), quien explica que en dicho marco la comprensión tiene un enfoque hacia las vivencias; de esta manera, el investigador busca el significado humano a un fenómeno social, como lo es el hecho de enseñar a investigar en la educación media, ya que se enfoca en indagar cómo los docentes entienden desde sus concepciones pedagógicas la importancia y la enseñanza de la investigación en el ámbito escolar. Esto, debido a que hay una significación particular desde su experiencia y su formación.

Particularmente, este diseño investigativo se enmarca dentro de la técnica del estudio de caso, enfocado en cómo, en conjunto, se enseña a investigar en la educación media del IPN, lo cual se analizará con la perspectiva de cómo los docentes, a partir de sus propias concepciones, enseñan la investigación desde las ciencias naturales, para, de esta manera, describir la corriente pedagógica en la que ellos trabajan y entender su postura desde su formación y su experiencia, además de la influencia que ello ejerce sobre el aprendizaje de sus estudiantes, entendiendo que la enseñanza de las ciencias naturales se ve enfocada desde la manera como los docentes enseñen a investigar, y entendiendo así la realidad de la educación media en este sentido para el IPN. Según este referente, cabe decir que los docentes participantes tenían diferentes formaciones académicas: uno tenía posgrado en la disciplina de biología, y otro, en educación (y ambos, con experiencia en el liderazgo de proyectos de carácter investigativo), mientras dos más tenían pregrado de licenciatura en física y biología y reivindicaban haber tenido formación en investigación y que su estrategia pedagógica era la búsqueda de textos guía.

Finalmente, permite describir y caracterizar el caso de estudio y lograr su interpretación y su comprensión generando los elementos que enmarcan su abordaje en las ciencias. Es así como se pretende, a partir de la revisión documental, el análisis de las concepciones y las prácticas de los docentes del IPN, así como generar conocimiento en torno a la investigación en ciencias naturales como estrategia de enseñanza en la educación media. Básicamente, esta investigación responde al cómo y al por qué de las prácticas y las concepciones docentes, para entender el fenómeno de interés, y, de esta manera, generalizar o inferir una teoría. El presente estudio es de tipo holístico -de una unidad única de análisis que comprende las concepciones de los docentes del área de ciencias naturales del IPN frente a la enseñanza de la investigación en la escuela- enmarcado en un caso único de tipo instrumental (Stake, 2005).

En esta investigación se hizo necesario aplicar entrevistas semiestructuradas a los docentes de educación media que enseñan investigación en el IPN y los de secciones como educación básica primaria y secundaria desde el área de ciencias naturales, los cuales fueron validados por docentes expertos en gramática, ciencias naturales 
y pedagogía. Por ello, esta investigación contempla el caso de los tres docentes del área de ciencias naturales del énfasis en educación media responsables de las asignaturas de tópicos en biotecnología, investigación, epistemología y asesores de línea de aplicaciones de la biofísica y aplicaciones de las ciencias naturales en la cotidianidad. Así mismo se procedió con los tres docentes adscritos al área de ciencias naturales de básica primaria y básica secundaria. En este sentido, se aborda no solo a los docentes que enseñan directamente la asignatura y asesorías desde la investigación escolar, sino a los que desde sus clases aportan a la formación investigativa de estudiantes en distintos niveles, lo cual permitió tener un análisis más amplio en torno a las concepciones del área frente a la investigación.

En segunda instancia, se abordó, a partir de la revisión documental, la fuente de información primaria desde los escritos de los docentes de educación media en torno a su enseñanza de la investigación escolar y secundaria desde los documentos de los proyectos realizados por los estudiantes, quienes han sido guiados en la investigación por dichos docentes. Cabe señalar que para tres docentes no se cuenta con escritos o proyectos guiados, por lo cual ellos aportan a la investigación en la consolidación de los lineamientos desde las entrevistas.

Para el análisis de la enseñanza desde el material elaborado por los estudiantes, se retoman diez proyectos realizados bajo la asesoría de los docentes; ello, a través de la revisión documental planteada por Alfonso (citado por Morales, 2003), donde resultan importantes la lectura y la escritura en la significación de los documentos y su interpretación, acorde al enfoque metodológico, por lo cual se utilizó como estrategia de procesamiento de datos el software ATLAS.ti, para análisis de información frente a los documentos y las entrevistas.

Las categorías planteadas responden a los interrogantes de esta investigación en tres ejes reconocidos en los objetivos de esta:

- Las concepciones pedagógicas docentes frente a la investigación. Dada la importancia de retomar las prácticas y las experiencias, tanto formativas como de liderazgo, de investigaciones de los docentes, las cuales enmarcan su manera de concebir la investigación.

- La importancia de la inclusión de la investigación escolar. Esta reconoce las bondades de incluir tal estrategia dentro del marco formativo de los educandos; en particular, en la educación media del IPN.
- Estrategias de enseñanza de las ciencias naturales. Esta categoría refiere dicha experiencia como acercamiento a la innovación en la enseñanza de las ciencias naturales en esos contextos escolares.

\section{Resultados}

Acorde con el paradigma interpretativo de la investigación cualitativa, se presentan los resultados obtenidos a partir de las entrevistas semiestructuradas y la revisión documental. Estos instrumentos permitieron el desarrollo de los objetivos, y arrojaron como resultado la descripción y la identificación de las corrientes pedagógicas que predominaron en los docentes enmarcados dentro de sus concepciones y sus prácticas. Al correlacionar los artículos trabajados, las entrevistas semiestructuradas y el análisis documental, se evidenciaron las categorías y se logró determinar puntos de encuentro de los docentes en una corriente pedagógica. Para el objetivo relacionado con la generación de los elementos pedagógicos desde la enseñanza de la investigación escolar en las ciencias naturales en educación media, se retomaron los estándares establecidos por el MEN para grados décimo y once en ciencias naturales, y los hallazgos con los docentes, buscando determinar, desde sus concepciones, estrategias para abordarla.

En la categoría concepciones docentes es importante tomar en cuenta que todos los participantes sustentaron el inicio de su formación como investigadores a partir de sus estudios de pregrado, y para algunos, como parte fundamental de los estudios posteriores que han llevado a cabo; sin embargo, se mencionan investigaciones de índole pedagógica, escolar y disciplinar en varios niveles, utilizando espacios alternativos para la ciencia y la tecnología, diseños experimentales, de campo y exploratorios, con el objetivo principal de ser aplicados en el área pedagógica. Ello demuestra que es muy importante para los estudiantes y los docentes utilizar el contenido de las investigaciones con un propósito social aplicable a la colectividad educativa de la cual hacen parte, y, de esa manera, contribuir al conocimiento como base para el mejoramiento de la comunidad. Además de ello, el grupo de docentes entrevistados considera que el proceso de investigación es esencial en pedagogía, pues posibilita el desarrollo de múltiples competencias cognitivas en los estudiantes, tales como la formación de un pensamiento crítico y la proposición de soluciones con el fin de solucionar un problema:

Es una herramienta orientada a la obtención de nuevos conocimientos para lo cual se requiere estrategias metodológicas para la solución de problemas o interrogantes de carácter científico o social. (Docente 2, comunicación personal, 8 de mayo de 2014) 
Es un proceso metódico que busca enriquecer el conocimiento que ha acumulado la humanidad hasta el momento, parte de los resultados de investigaciones previas en pro de enriquecerlas o darles nuevos rumbos, en el marco de la rigurosidad y la objetividad. (3:32. Docente 3, comunicación personal, 2 de mayo de 2014).

Esta mirada en torno a la investigación, evidenciada en las entrevistas y comparada en la revisión de documentos realizados por los docentes, permite evidenciar la tendencia de la concepción de los docentes, centrada en el reconocimiento de características propias de la corriente pedagógica positivista, enmarcada dentro de la experimentación, el desarrollo del método científico, el análisis cuantitativo de datos a partir de la medición, aplicaciones de las diferentes disciplinas de las ciencias naturales y procesos de análisis objetivos y mecánicos hacia la búsqueda de un conocimiento científico. Sin embargo, se evidencia en algunos docentes el abordaje de aspectos de un enfoque interpretativo hacia las relaciones con datos cualitativos que incorporan aspectos sociales, los cuales buscan que desde la investigación disciplinar se generen impactos en la comunidad. Esto, a su vez, manifestando un interés en que la investigación científica permee costumbres y comportamientos.

En la categoría investigación escolar, los docentes manifiestan y reflejan en sus escritos y sus proyectos asesorados la importancia de la investigación como método pedagógico para indagar, innovar, ampliar conocimientos, experimentar estrategias o alternativas en el desarrollo de las prácticas de aprendizaje en el aula, donde la mayoría de los registros analizados reflejó la forma como los procesos de investigación están ligados a la profundización del conocimiento de un tema o al hallazgo de la solución de un problema, cuya aplicación, por lo general, implica una intención de índole social.

Otro aspecto para resaltar en la mayoría de los proyectos fue que se refirió el método cuantitativo de tipo experimental como la principal manera de conseguir la información, ejemplificada mediante entrevistas, encuestas, muestras y revisión documental. El proceso cualitativo fue secundario frente al cuantitativo, y algunos reivindicaron la utilización de un método mixto como forma de procesamiento de datos. Este procedimiento es entendido tanto para el conteo de estadísticas y la descripción de las propiedades de las muestras como para el uso de preguntas cerradas y abiertas.

Algunos docentes reconocen el proceso investigativo como un instrumento pedagógico que debería ser implementado desde la niñez para lograr un mayor beneficio académico y el desarrollo de las competencias cognitivas que son necesarias en los estudiantes para llevar a cabo los procesos de investigación desde el aula de clase, por lo cual son indiscutibles los beneficios académicos, sociales, interpersonales y laborales que se obtienen con la práctica de las competencias requeridas en un proceso de investigación:

En mi opinión lo más importante es delimitar una problemática objetivo a la cuál dirigir la investigación, para así, tener una idea de hacia dónde está dirigida la investigación, cuáles van a ser los resultados esperados de la misma y la aplicabilidad que pueda tener dentro de un contexto. (Docente 3, comunicación personal, 7 de mayo de 2014).

Una característica importante para esta investigación es que para los docentes es esencial seguir las pautas que dicta la ejecución del método científico para llevar a cabo una investigación. Los procesos cognitivos que parten del planteamiento de preguntas y buscan que el estudiante reflexione, analice e interprete son la guía básica que conducirá al investigador a formular hipótesis con el fin de encontrar soluciones específicas.

Para los docentes, la investigación permite una forma de aprendizaje libre, que parte de cuestionamientos propios de los mismos estudiantes desde su etapa infantil, sin represiones, para estimular la exploración de los contenidos que les puedan interesar, $\mathrm{y}$, de tal manera, utilizar el método investigativo básico de hacer preguntas para adquirir conocimiento, por lo cual tiene diversos beneficios pedagógicos y cognitivos para los estudiantes, no solo en cuanto al estudio de un tema específico, como lo son las ciencias naturales, sino mediante el tratamiento de los procesos que se llevan a cabo durante la experimentación. Para los docentes, es indiscutible que, durante el transcurso del aprendizaje a través de la investigación, los estudiantes conocen y vivencian de forma práctica las etapas que componen el desarrollo del método científico: "Lo más importante de investigar es que la investigación se convierta en una herramienta que permita avanzary explorar en los conocimientos, permita establecer contacto con la realidad y responda a interrogantes cotidianos" (3:25. Docente 2, comunicación personal, 8 de mayo de 2014).

En la categoría enseñanza de las ciencias naturales, al acercar a los estudiantes a diversas materias de estudio se amplía la visión del conocimiento que poseen, de forma que se estimula su curiosidad respecto a los contenidos que les pueden interesar, por lo cual prevalece la importancia del tratamiento de temáticas relacionadas con el medio ambiente, pues les ayuda a los sujetos a comprender y a estimar el valor de los seres vivos, por 
lo cual es posible percibir cómo las actividades que el docente puede ejercer en el aula de clase dependen, en gran medida, del espacio y el tiempo académicos en el que se desenvuelve, al igual que de su creatividad como pedagogo, de los recursos de los cuales puede disponer, del contenido que abordará, y, así, construir las estrategias que posibiliten la mejor manera de conseguir resultados; es esta una manera muy pertinente de acercar a los educandos a la ciencia desde el método científico y su cotidianidad. "En Ciencias se permite desarrollar diseños experimentales basados en el método científico, estudios de caso para indagar, problematizar y formular hipótesis, y en el quehacer pedagógico desarrollar nuevas estrategias para facilitar el aprendizaje" (Docente 2, comunicación personal, 8 de mayo de 2014).

Como código emergente, se evidencia el beneficio de emplear el proceso investigativo como estrategia pedagógica para fomentar y extender el interés de los alumnos por este tipo de tópicos y de metodología, considerando dicho interés fundamental para facilitar el estudio de esa área del conocimiento. Ello, evidenciado en la importancia de captar los intereses y las motivaciones de los estudiantes hacia la ciencia y de poder relacionar sus aprendizajes con la cotidianidad, al entender que los proyectos que se tomaron para el presente estudio, asesorados por los docentes, tenían como motivante las ideas y las inquietudes de los educandos, lo cual hacía que ellos mismos los desarrollaran, con el fin de responder a sus inquietudes.

Considero que debe ser lo esencial para enseñar las ciencias naturales en los estudiantes; ya que, una de las rutas para transformar la escuela y las prácticas pedagógicas en ciencias, se fundamenta en la disposición intelectual y critica de los agentes principales de la educación "los docentes", ya que, somos nosotros los tejedores de comunidades de aprendizaje. Con el ejercicio de la investigación como práctica pedagógica, abrirá horizontes tanto a los estudiantes en las nuevas formas de interpretar el mundo y plantear soluciones; así como la apertura de la brecha de la educación y sus prácticas, en este mundo globalizado y competitivo. (Docente 6, comunicación personal, 13 de mayo de 2014).

En relación con los hallazgos reportados desde los análisis de la presente investigación, retomando los estándares básicos en competencias en ciencias naturales planteados por el MEN y con base en la validación hecha por la jefatura de área y dos docentes del área de ciencias naturales, se proponen lineamientos para el abordaje de la investigación escolar, los cuales se ajustan a los requerimientos y las oportunidades que desde el PEI ofrece el IPN a la formación de sus estudiantes.

\section{Desde los estudiantes}

- Fomentar el desarrollo de competencias argumentativas, interpretativas y propositivas, a partir de la aplicación y el análisis de fenómenos científicos desde la investigación escolar.

- Seleccionar información desde diversas fuentes, para conceptualizar y construir procesos investigativos.

- Aportar al desarrollo de la autonomía y la crítica en los diferentes campos del saber de las ciencias naturales.

- Desarrollar una visión de ciencia como eje transformador de conocimiento.

- La investigación escolar debe verse como una estrategia para profundizar en el conocimiento.

- La investigación fomenta la postura crítica y argumentativa frente a la explicación de fenómenos.

- Involucrar a los estudiantes en el desarrollo social de su comunidad, con el fin de hacerlos participes de su entorno.

\section{Desde los docentes}

- Relacionar diferentes disciplinas en la explicación y la interpretación de fenómenos naturales.

- Buscar que el estudiante se apropie de su aprendizaje desde la motivación y la búsqueda del conocimiento a partir de la investigación.

- Construir, transformar y generar nuevas prácticas pedagógicas y sociales a partir de la investigación.

- La investigación debe buscar el análisis de situaciones desde la exploración de fenómenos, a partir de métodos establecidos de manera rigurosa.

- Buscar generar investigaciones escolares que partan de las motivaciones y los intereses de los estudiantes.

- Guiar de manera sistemática los procesos investigativos propuestos por los estudiantes tomando en cuenta elementos constitutivos de la investigación formal y pura.

\section{Desde las instituciones}

- Socializar y comunicar desarrollos adelantados a partir del ejercicio investigativo tanto de los estudiantes como de los docentes.

- La investigación debe verse como una estrategia que permite indagar, innovar, profundizar en conocimientos, experimentar y generar nuevos aprendizajes.

- Apoyar el trabajo investigativo de los docentes y los estudiantes desde incentivos y desde la generación de espacios académicos. 


\section{Desde la enseñanza de las ciencias}

- Acercar a los estudiantes, desde la investigación, a conocimientos y a métodos científicos.

- Desarrollar competencias científicas e investigativas en los estudiantes a partir de la indagación y el proceso metódico de la investigación.

- Aplicar conceptos y procesos científicos en contextos cotidianos.

\section{Conclusiones}

Este trabajo resultó pertinente en la medida en que a través del estudio de caso en la educación media del IPN (particularmente, en el énfasis en ciencias naturales y enmarcado dentro de la revisión documental), se establecieron las concepciones pedagógicas de los docentes sobre la investigación, pues se logró un acercamiento a su definición en el contexto particular, y se reconoció cómo desde la formación y la experiencia de los docentes esta se trabaja en la educación media a partir de proyectos y en el abordaje de diferentes temáticas. Además de ello, se da a conocer la necesidad de la investigación en la escuela y su relación con la enseñanza de las ciencias naturales aportando elementos pedagógicos específicos que se siguen al enseñarla y siendo escenario educativo de gran importancia para el país, dada su naturaleza de centro de innovación e investigación pedagógica y didáctica, desde un análisis de aspectos teóricos y prácticos en torno a este enfoque investigativo en la educación media y la enseñanza de las ciencias naturales.

Para el caso del primer objetivo específico, el cual buscaba describir las concepciones pedagógicas que sobre investigación tienen los docentes de educación media del IPN a partir de su formación y de su experiencia pedagógica en la enseñanza de las ciencias naturales, se hizo evidente la relación entre la forma como el docente aprendió a investigar y la forma como enseña; esto, a su vez, relacionado con su formación y su experiencia en los campos disciplinar y pedagógico, y encaminando las investigaciones de los estudiantes hacia el ejercicio científico desde el saber de su disciplina. Dicho aspecto permite reconocer diferencias entre los docentes que han desarrollado investigaciones y quienes se guían en el proceso investigativo por manuales y textos, pues, de cierta manera, su concepción de investigación se ve un poco limitada a desarrollos empíricos y poco profundos en torno al conocimiento.

La tendencia de las concepciones pedagógicas de los docentes frente a la investigación escolar se enfoca hacia las corrientes que permiten comprender y dimensionar las maneras como estos han dirigido sus prácticas y encaminan sus desarrollos didácticos; ello, como lo plantea
Ocampo (citado por López, 2012), sugiere una identificación particular en las prácticas docentes, un valor y un significado concretos, lo que se evidenció en el discurso específico dado por cada docente a la hora de hablar de investigación y de su incorporación en la enseñanza de las ciencias, enmarcando los intereses de cada individuo, permitiendo correlacionar posturas y prácticas para el área de ciencias naturales (Padrón, 2007). La corriente que encierra estas concepciones pedagógicas de los docentes se caracteriza por la tendencia positivista, que se enmarca dentro de investigaciones disciplinares y cuantitativas, viendo la ciencia desde hechos corroborables y enmarcados en un proceso detallado y estadístico a partir de un análisis objetivo de un fenómeno, y delimitado ello por la aplicación del método científico. Sin embargo, se empieza a incluir la corriente pedagógica interpretativa y crítico-social, al socializar los hallazgos cuantitativos obtenidos procurando generar transformaciones sociales, desde el campo cultural y de intervención con la comunidad.

Los docentes en su concepción de investigación reconocen los beneficios académicos, sociales, interpersonales y laborales que se obtienen con la práctica de las competencias requeridas en un proceso de investigación, desde la generación y la profundización del conocimiento, el seguimiento riguroso de procesos metódicos en la búsqueda de información y la recolección de datos, la interpretación y la argumentación frente a fenómenos y el desarrollo crítico y propositivo desde su realidad y su cotidianidad; todo eso lleva al estudiante a ser un actor de procesos de transformación desde su motivación y su interés, lo cual es muy acorde con lo propuesto por Tamayo (1987) en torno a las bondades de la investigación, al hablar de métodos sistemáticos basados en el método científico que buscan profundizar y generar conocimiento.

Dado el surgimiento de la categoría emergente enfocada en la importancia de abordar la investigación generada a partir de los intereses y las motivaciones de los estudiantes, dicho aspecto se debe tener en cuenta a la hora de proponer líneas y ejes temáticos que desde la institución se planteen para el desarrollo de la investigación.

Bajo las concepciones pedagógicas de los docentes, y respondiendo al segundo objetivo, que era identificar las prácticas pedagógicas de los docentes de ciencias naturales, en torno a la investigación escolar desde los trabajos realizados por estudiantes de educación media del IPN, se evidenció cómo la investigación permite acercar a los estudiantes a la ciencia mediante la aplicación del método científico; específicamente, desde la experimentación y desde el hecho de potenciar habilidades investigativas partiendo del campo científico, y que son entendidas como "el dominio de las acciones generalizadoras del método científico 
que potencian al individuo para la problematización, teorización y comprobación de su realidad profesional, lo que contribuye a su transformación sobre bases científicas" (Paulo, 2012, p. 15). Además, se fortalecen el acercamiento y la reflexión hacia el entorno vivo, el ambiente y sus relaciones con la sociedad desde la congregación de diversas disciplinas en el proceso investigativo. Otro aspecto para tener en cuenta es la posibilidad de, a partir de la investigación, adoptar estrategias pedagógicas y didácticas que profundicen en el conocimiento y su aplicación en la cotidianidad. Esto se ve evidenciado en el desarrollo de los documentos analizados, que, en su mayoría, mostraron de forma tangible aplicaciones de la investigación escolar en diferentes fenómenos y contextos, los cuales hicieron que los estudiantes profundizaran y generaran conocimiento.

Otro aporte desde la identificación de estas prácticas investigativas en los docentes radica en entender la investigación como base de la formación docente y de su ejercicio, como lo propone Marín (2012), pues, siguiendo a este autor, es indispensable recalcar la necesidad y la importancia de que el docente no solo sea el facilitador del conocimiento, sino también, el investigador de su propia práctica y su actividad profesional y disciplinar, pues en el ejercicio pedagógico no debe limitarse a un único método o rechazar las estrategias empiristas, sino que debe valerse del dinamismo del conocimiento científico y la importancia del trabajo en colectivo.

\section{Referencias}

Aldana, G. (2008). Enseñanza de la investigación y epistemología de los docentes. Cundinamarca: EDUC.

Briones, G. (2002). Epistemología de las ciencias sociales. Bogotá: Arfo Editores.

Cañal, P. (2007). La investigación escolar. Didáctica de las Ciencias Experimentales, 9-19.
Colciencias (2012). La investigación como estrategia pedagógica (IEP). Recuperado el 8 de febrero de 2014, de Colciencias http://www.colciencias.gov. co/wiki_ondas/la-investigaci-n-como-estrategia-pedag-gica-iep

Gorodokin, I. (2005). La formación docente y su relación con la epistemología. Revista Iberoamericana de Educación. ISSN 1681-5653

Herrán, A., Hashimoto, E., \& Machado, E. (2005). Investigar en educación. Fundamentos, aplicación y nuevas perspectivas. Madrid: Dilex.

López, J. (2012). Las corrientes pedagógicas en la educación colombiana. Revista Hechos, p. 61.

Marín, J. (2012). La investigación en educación y pedagogía. Bogotá: USTA.

MEN (2012). Recuperado el 10 de octubre de 2013, de http://www.mineducacion.gov.co/1621/w3-article-246098.html

Morales, O. (2003). Fundamentos de la investigación documental y la monografía. En: Norelkys Espinoza y Ángel Rincón (Eds.). Manual para la elaboración y presentación de la monografía. Mérida, Venezuela: Universidad de Los Andes

Padrón, J. (2007). Tendencias epistemológicas de la investigación científica en el siglo XXI. Cinta Moebio. (28), 1-32. ISSN 0717-554X.

Paulo, M. (2012). El desarrollo de habilidades investigativas en las Universidades de Ciencias Pedagógicas de Cuba y Bié (Angola). Revista Congreso Universidad, I (2), 1-13. ISSN: 2306-918X.

Stake, R. (2005). Investigación con estudios de caso. Madrid: Morata.

Tamayo, M. (1987). Aprender a investigar. En: M. Tamayo. Aprender investigando (Módulo 2). Bogotá: ICFES. 